\title{
The structural stability and biophysical properties of the mega-protein Erythrocruorin is regulated by polyethylene glycol surface coverage
}

Chintan Savla ${ }^{1}$ Andre F. Palmer ${ }^{*}$

${ }^{1}$ William G. Lowrie Department of Chemical and Biomolecular Engineering, The Ohio State University, Columbus, $\mathrm{OH}, 43210$

Grant Numbers: NIH R01 HL138116， R01HL126945, and R01EB021926.

*Correspondence: Andre F. Palmer, William G. Lowrie Department of Chemical and Biomolecular Engineering, The Ohio State University, 452 CBEC, 151 West Woodruff Avenue, OH 43210, USA. E-mail: palmer.351 dosu.edu. Phone: (614) 292-6033. 


\section{Supplemental Information}
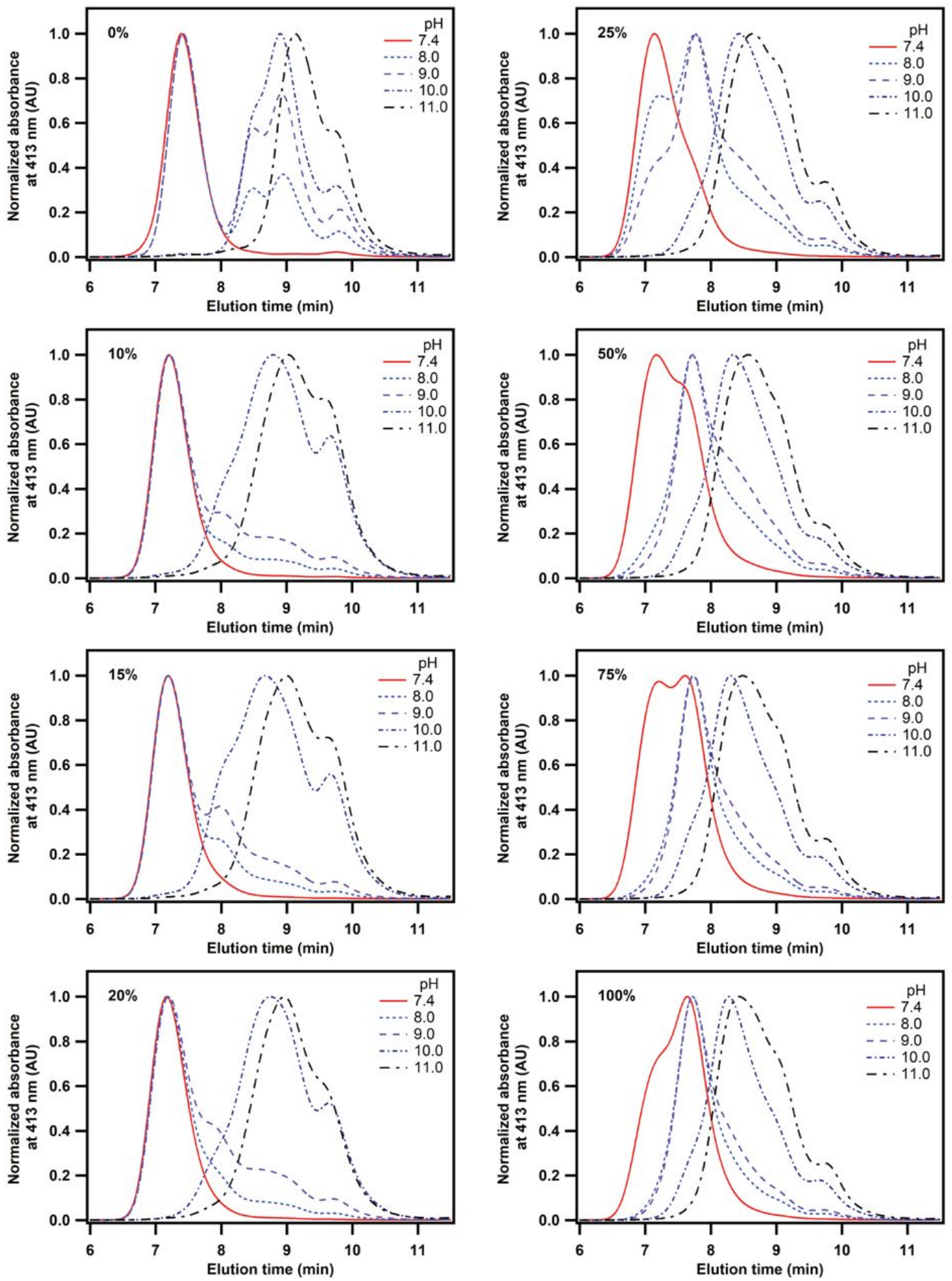
Figure S1: Dissociation of PEG-LtEc species with change in $\mathrm{pH}$ from 7.4 to 11.0. LtEc is known to dissociate at $\mathrm{pHs}>8.0$. Partially PEGylated structures have improved structural stability, whereas densely and over-PEGylated structures dissociate easily at pH 8.0 and above.

(A)

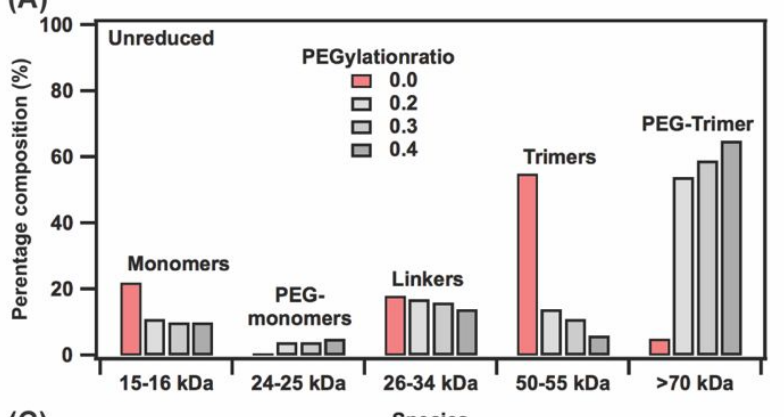

(C)

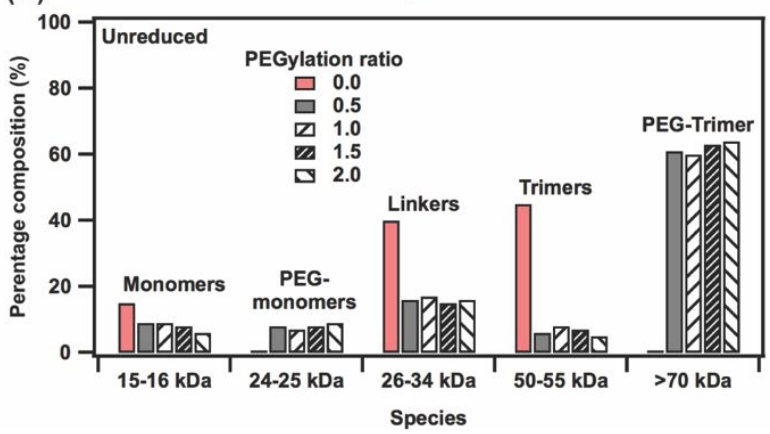

(B)

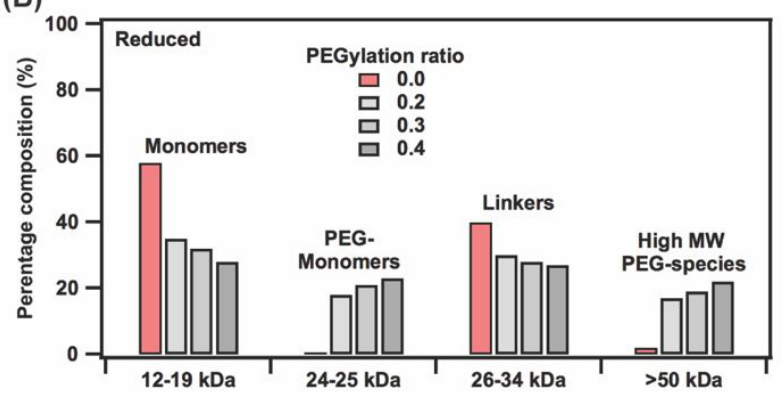

(D)

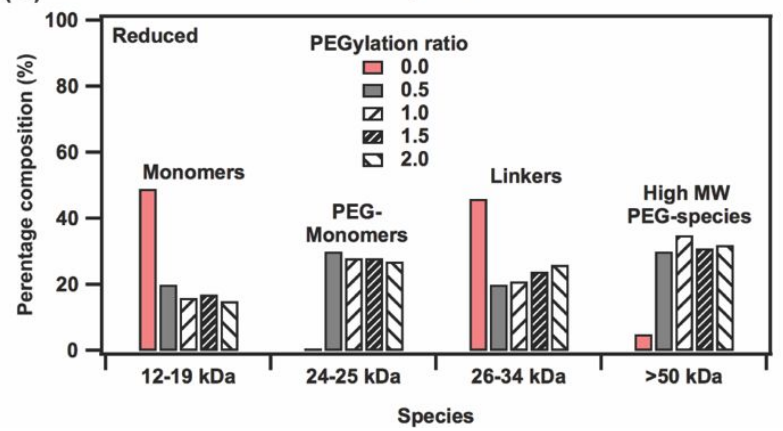

Figure S2: Densitometric analysis of SDS-PAGE runs of LtEc and PEG-LtEc species. There was a decrease in the composition of monomer and trimer species with increasing PEGylation ratio along with the appearance of PEG-monomer and PEG-trimer species confirming successful PEGylation. 